\title{
Acute kidney injury in SARS-COV-2 infection
}

\author{
Oswaldo Jesus Rodrigues da Motta ${ }^{1}$, Jaqueline Machado da Fonseca ${ }^{2}$, Isadora de Carvalho e Silva ${ }^{2}$, Flávio Marques Andreon ${ }^{1}$, \\ Lorena Souza e Silva ${ }^{2}$, Paulo Sérgio Balbino Miguel ${ }^{1,2}$, Andréia Patrícia Gomes ${ }^{2}$ \\ ${ }^{1}$ Department of Medicine and Nursing, Universidade Federal de Viçosa (UFV), Viçosa, Brazil \\ ${ }^{2}$ School of Medicine at Faculdade Dinâmica do Vale do Piranga (FADIP), Ponte Nova, Brazil
}

\section{ABSTRACT}

The current pandemic of COVID-19 - Coronavirus Disease-19, caused by the pathogen SARS-Cov-2, responsible for thousands of deaths in 2020 , has mobilized the scientific community, who aim to understand the different aspects of the disease and propose new treatments and prevention modalities. Research in several areas is evaluating the extent of infection and kidney disorders related to the virus. The aim of this study is to describe the effects of COVID-19 on the renal system. Final considerations point to the main complications of the effects of infection and renal disorders caused by COVID-19, such as acute kidney injury and a consequent increase in the disease's poor prognosis.

Keywords: Kidneys, Kidney disorders, COVID-19, SARS-CoV-2.

\section{INTRODUCTION}

Coronavirus is a pathogen that infects vertebrates and was originally discovered in birds during the 1930s. Furthermore, it is known that there are seven different types of this virus that can infect humans, possibly leading to respiratory, gastrointestinal, neurological, and/or liver disease ${ }^{1}$. Within these, two are noteworthy, the first is SARS-Cov-2, which is responsible for the current COVID-19 pandemic, and can cause severe acute respiratory syndrome (SARS), and the other is MERS-COV, a virus responsible for Middle East respiratory syndrome (MERS) ${ }^{2}$.

During the first week of January 2020, the Chinese government reported the presence of a new type of coronavirus, not yet known, that was later named SARS-CoV-2 on February 11th of the same year. Ever since, the World Health Organization (WHO) has been diligently working, alongside with various scientists to decipher this new virulent pathogen with the objective of controlling its spread and eventually finding a protective method to rid the population of the virus, whether it be through treatment or vaccination ${ }^{2}$.

The COVID-19 disease is transmitted from person-to-person, primarily through the inhalation of respiratory droplets produced when an infected person sneezes, coughs, or even just verbalizes. These small droplets can also contaminate surfaces, which when touched by another individual who may, eventually, touch his or her eyes, mouth or nose, can also cause an infection by the SARS-CoV-2 virus. In addition, it is imperative to note that asymptomatic individuals who are infected can also spread the microorganism to others ${ }^{2}$.

The carriers of SARS-CoV-2 can be asymptomatic, manifest few symptoms, which can be easily confused with those caused by the common cold, for example, or develop more serious symptoms that, in some cases, can lead to death ${ }^{3}$. Among the severe symptoms that can result in morbidity and death in patients infected with SARS-CoV-2 is acute kidney injury (AKI), responsible for damaging the function of this organ ${ }^{3}$.

This injury can result from direct infection by the virus or secondary inflammation ${ }^{4}$. The incidence of AKI in these patients is high, reaching $29 \%$, in patients with severe pneumonia caused by SARS-CoV-2. In addition, the mortality rate has been shown to be much higher in patients with acute kidney infection than those who do not have this type of infection ${ }^{5}$. However, despite the importance of acute kidney infection in patients with COVID-19, there is a need for further studies involving renal care in these patients.

The progression of the disease caused by SARS-CoV- 2 may give rise to a loss of homeostasis throughout the body, leading to a generalized immune response to infection, affecting various organs and organ systems. Within the systemic complications that can be caused by the COVID-19 virus, renal injuries are of high importance due to the high mortality rate within infected patients that manifest acute kidney injury (AKI) and cardiorenal syndrome. Furthermore, studies have reported that during certain situations, such as electrolyte imbalance and hypertension, the virus can attack the renal cells directly, raising the risk of morbimortality of this pathological process ${ }^{6}$. Therefore, the purpose of this review is to analyze the AKI caused by the SARS-CoV-2 in addition to offering new suggestions for prevention and treatment.

\section{SARS COV-2 AND ACUTE KIDNEY INJURY}

The presence of a moderate to severe acute kidney injury (AKI) poses an important risk to patients with acute respiratory distress 
syndrome (ARDS), which results in a significant increase of the patient's mortality risk. In patients with ARDS, the AKI can be associated with numerous causes, such as gas exchange deficiencies, hemodynamic instabilities due to right-sided heart failure (RHF) and fluid overload.

The immune mediated inflammatory response plays an important role in the onset of $\mathrm{AKI}$, as a result of the large quantity of certain inflammatory mediators released in the bloodstream with the ability to interact with kidney cells, leading to endothelial dysfunction, microcirculatory instability and tubular injury. Moreover, studies have shown that the beta coronavirus, SARS-CoV, and the newfound coronavirus, SARS-CoV-2, use the angiotensin-converting enzyme 2 (ACE2) as a receptor, and after binding to this receptor, enter the host cells. ACE2 can be found on the surfaces of tubular renal cells and if this tissue becomes infected, a local inflammatory response can arise, prompting the occurrence of $\mathrm{AKI}$ in patients.

In patients with ARDS and AKI, the latter manifests itself on average 9 days upon hospital admission, along with secondary infections and acute heart injury. Some of the risk factors which increase the odds of developing AKI that stand out are age, diabetes, the severity of illness and a history of cardiorenal syndrome (CRS). Accordingly, it is of utter importance that the risks of patients with SARS-CoV-2 who develop severe AKI be determined, especially those in need of renal replacement therapy, so that preventative and protective measures may be provided. Such measures include preliminary examinations by nephrologists, checking creatinine blood levels, urinary output, hemodynamics, and, when possible, replacing the procedures that use iodinated contrast or nephrotoxic medications with less harmful options ${ }^{7}$.

\section{RESULTS AND DISCUSSION}

The probable paths that lead to kidney injury because of the presence of COVID-19 include direct kidney infection, as well as aggressions that are caused by the cascade of cytokines linked to sepsis. This direct renal aggression occurs due to the existence of the angiotensin-converting enzyme 2 in the proximal renal tubules, which is a receptor that allows the SARS-COV-2 virus to enter the target cell.

In a study assessing the state of patients undergoing maintenance hemodialysis (MHD) in the context of the COVID-19 pandemic, addressing strategies to reduce the spread of the disease in outpatient hemodialysis facilities ${ }^{8}$, it was found that most existing research data was obtained from the clinical course of Chinese and Italian patients and from patients with MHD. The research is in the form of preliminary reports, which makes it difficult to accurately describe the incidence and the impacts of COVID-19 in these patients. Another topic critically addressed in this study is the standardization of diagnostic criteria.

In light of the current scenario, explanatory documents were formulated, aiming to prevent and control the spread of COVID-19 in hemodialysis clinics. These documents address the education of patients and staff regarding preventive measures and emphasize the need to prepare hemodialysis services for the possible increase of incoming suspected and confirmed patients, in addition to preparing the environment to avoid contamination.

Patients contaminated with COVID-19 in need of hemodialysis were treated with continuous renal replacement therapy or high-volume hemofiltration with absorbent membranes to eliminate inflammatory cytokines. There were direct contact limitations, with only two patients being treated at a time, which, with the increase in demand, required the establishment of an exclusive space for patients in quarantine. Patients who had received transplants and underwent peritoneal dialysis received home care to minimize visits to hospitals.

Acute kidney injury occurs more commonly in patients with severe pathologies, especially those admitted to the ICU. It is known that the storm of inflammatory cytokines linked to the innate immune response is associated with the damage to renal tubules that involves hypoperfusion. In addition to this, studies show that there is a direct effect of SARS-CoV-2 on renal cells, expressing serine protease, which play a fundamental role in viral uptake and are highly expressed in the presence of the COVID-19 virus in various cells, such as podocytes and epithelial cells renal tubules. This can lead to albuminuria and hematuria. Such conditions associated with the isolation of virus RNA in renal biopsy corroborate the theory that the virus has a certain tropism towards the kidney.

Another study ${ }^{9}$ aimed to identify a possible explanation for the mechanism of how acute kidney injury progresses in COVID-19 infection. The analysis of normal renal cells was performed, observing the location of the ACE2 and TMPRSS genes, showing an important coexpression in podocytes and in the proximal rectum tubule. The TMPRSS2 gene, acting as an important mediator of SARS-CoV-2 when entering cells, was co-expressed with the podocyte ACE2 gene. From this, it was noted that Western donor cells stood out over Asian cells, suggesting that this population has a higher risk of developing AKI in the context of COVID-19.

Likewise, in a comparison of the expression of the ACE2 and TMPRSS genes in cells of other organs such as lungs, esophagus, small intestine, colon and kidney, there seemed to be no difference in these genes' expression, indicating that the kidney is the target organ of SARS-CoV-2. Finally, this study concluded that the podocytes and proximal straight tubules, which play an important role in filtration, excretion and reabsorption of urine, are particularly susceptible to the effects of viruses and bacteria.

Such an occurrence induces massive proteinuria, which tallies with what was seen in the analysis of patients infected with SARS-CoV-2, especially those who progressed to AKI, proven to possess proteinuria. Additionally, recent studies show the presence of SARV-CoV-2 in urine samples from severely infected patients and explain that the entry of the virus into the bloodstream, itself, can lead to AKI, contributing to the hypothesis that this virus has renal tropism.

Due to the constant spreading of SARS-CoV-2 and its high morbidity and mortality rate, it is essential to consider the possible scarcity of resources, taking into account the existing risk of patients with renal failure not having access to hemodialysis services. Therefore, the formulation of contingency plans becomes indispensable. 


\section{FINAL CONSIDERATIONS}

In this article we reviewed the main findings surrounding the effect of SARS-CoV-2 on kidney diseases, such as AKI, and discussed the clinical treatment of patients infected with COVID-19, who did, or did not, have a chronic kidney disease. Recent researches shows that ACE2 is also expressed in organs such as the kidneys, heart and intestines, which supports the hypothesis that SARS-CoV-2 may have tropism towards these organs, affecting the body's homeostasis and contributing to the spread of the pathogen throughout the organism.

With regard to the factors of poor prognosis and death of patients with renal comorbidities infected by COVID-19, patients with advanced age, multiple comorbidities and who were admitted with critical clinical conditions succumbed quicker to death. Moreover, AKI is evidently a common complication among these patients, seeing as the injury was found in $25 \%$ of those who died from SARS-CoV-19. Based on studies with renal biopsy in patients infected with SARS-CoV-2, the question of whether there is a greater risk of African American patients who are infected with the virus developing kidney disease, linked to the APOL1 gene, must be raised.

Current studies of viral nucleocapsid proteins, on site and post-mortem, have found that the SARS-CoV-2 antigens are concentrated in renal tubules, suggesting that this virus directly infects the human kidney, causing the spread of the virus throughout the body. So far, it is known that diabetic and hypertensive patients are part of the SARS-CoV-2 risk group. Since most of these patients use angiotensin receptor blockers (ARBs), there is a hypothesis that this drug is predisposing these individuals to infection by COVID-19 by increasing the expression of ACE2 in various organs, such as the kidneys and heart, and possibly in lung cells as well.

However, some research shows that a substantial growth in the probability of a positive test for COVID-19 was not found among patients who tested positive in association with five common classes of antihypertensive drugs, including ARBs and ACE inhibitors. Despite the lack of consistent data encompassing the impact of COVID-19 on patients with chronic kidney disease, transplanted patients and dialysis patients, there is a conviction that AKI is an important factor for a poor prognosis in patients infected by the virus. Further studies and guidelines for the management of dialysis centers are necessary, reinforcing safety measures needed to avoid the transmission and contaminations of patients who use this service and who, in general, are mostly immunodeficient.

Disclosure of potential conflicts of interest: none declared

\section{References}

1. Cespedes MS, Souza JCRP. SARS-CoV-2: uma revisão para o clínico. Publica em Abril de 2020 Disponivel em: < https://preprints.scielo.org/index.php/scielo/preprint/download/26/41/35>. Acesso em: 27 de abril de 2020

2. Organização Pan-Americana da Saúde - OPAS Brasil. Folha informativa -COVID-19 (doença causada pelo novo coronavirus). 27 de abril de 2020. Disponível em: < https://www.paho.org/ bra/index.php?option $=$ com_content\&view=article\&id=6101: covid19\& $\mid$ temid $=875>$. Acesso em: 27 de abril de 2020

3. Moitinho MS, Belasco AGS, Barbosa DA, Fonseca CD. Acute Kidney Injury by SARS-CoV-2 virus in patients with COVID-19: an integrative review. Rev Bras Enferm. 2020;73(Suppl 2): 1-8.

4. Joseph A, Zafrani L, Mabrouki A, Azoulay E, Darmon M. Acute kidney injury in patients with SARS-CoV-2 infection. Ann. Intensive Care. 2020;10(1):1-8.

5. Soleimani M. Acute kidney injury in SARS-CoV-2 infection: direct effect of virus on kidney proximal tubule cells. Int J Mol Sci. 2020;21(9):3275.

6. Cespedes MS, Souza JCRP. SARS-CoV-2: uma revisão para o clínico. Publica em Abril de 2020. Disponível em: < https://preprints.scielo.org/index.php/scielo/preprint/download/26/41/35>. Acesso em: 27 de abril de 2020

7. Fanelli V, Fiorentino M, Cantaluppi V, et al. Acute kidney injury in SARS-CoV-2 infected patients. Crit Care. 2020;24(1):155

8. Ikizler TA. COVID-19 and dialysis units: what do we know now and what should we do? [published online ahead of print, 2020 Mar 23]. Am J Kidney Dis. 2020; Jul;76(1):1-3.

9. Pan XW, Xu D, Zhang H, Zhou W, Wang LH, Cui XG. Identification of a potential mechanism of acute kidney injury during the COVID-19 outbreak: a study based on single-cell transcriptome analysis [published online ahead of print, 2020 Mar 31]. Intensive Care Med. 2020 Jun;46(6):1114-1116.

\section{Correspondence to:}

Oswaldo Jesus Rodrigues da Motta, PhD

Department of Medicine and Nursing

Universidade Federal de Viçosa (UFV)

Av. Peter Henry Rolfs, s/n Campus Universitário

Viçosa - MG, 36570-900, Brazil

E-mail: oswaldo.motta@ufv.br 